J. Lake Sci. (湖泊科学) , 2011, 23(2): 209-216

http: //www. jlakes. org. E-mail : jlakes@niglas.ac.cn

(C) 2011 by Journal of Lake Sciences

\title{
高矿化度沙漠湖泊水体的光学特性"
}

\author{
巫文文 ${ }^{1}$, 关洪军 ${ }^{1}, \mathbf{E}$ 洪涛 $^{2}$, 敖春来 $^{3}$ \\ ( 1 : 解放军理工大学工程兵工程学院, 南京 210007) \\ (2: 中国科学院南京地理与湖泊研究所湖泊与环境国家重点实验室,南京 210008) \\ (3: 宁夏军区给水团,银川750021)
}

\begin{abstract}
摘 要: 沙漠湖泊水体和东部大型湖泊如太湖等存在较大差异. 为深人研究沙漠湖泊水体的光学特性, 利用腾格里沙漠 月亮湖实测水面 ASD 高光谱数据和同步采集的水样, 对水体矿化度与叶绿素 $\mathrm{a} 、$ DOC、悬浮物的含量进行相关性分析, 比 较沙漠湖泊水体和其它水体的黄色物质、浮游植物、非藻类悬浮物吸收特征光谱, 探讨不同波段中水深和矿化度对水体 反射率的影响. 结果表明水体矿化度和水深分别是影响沙漠湖泊水体固有光学特性和表观光学特征的最主要因素: 矿化 度显著影响所有物质的吸收特性, 与 DOC 含量和 $240-300 \mathrm{~nm}$ 段内的黄色物质吸收系数具有很好的相关性; 水深显著影 响水体的遥感反射率,但在 $550-700 \mathrm{~nm}$ 波段同一水深范围内矿化度成为影响水体反射率的主要因素. 本研究为今后利用 遥感数据监测沙漠湖泊矿化度等水质指标提供了理论基础.
\end{abstract}

关键词: 沙漠湖泊; 矿化度;月亮湖;腾格里沙漠; 吸收系数

\section{Water optical properties of high mineralization in desert lakes}

\author{
WU Wenwen ${ }^{1}$, GUAN Hongjun ${ }^{1}$, DUAN Hongtao ${ }^{2} \&$ AO Chunlai $^{3}$ \\ (1: Engineering Institute of Engineer Corps, PLA Univ. of Sci. \& Tech, Nanjing 210007, P. R. China) \\ (2: State Key Laboratory of Lake Science and Environment, Nanjing Institute of Geography and Limnology, Chinese Academy \\ of Sciences, Nanjing 210008, P. R. China) \\ (3: Water Supply Engineering Support Corps in Ningxia Military Area, Yinchuan 750021, P. R. China)
}

Abstract: Lakes in desert areas are very different from large lakes in the eastern part of China, like Lake Taihu. The objective of this study was to find out the optical properties of desert lakes. Reflectance spectra were measured in October 2009 with ASD FieldSpec spectrometer. Concurrently, water samples were collected directly from the lake to be analyzed in laboratory. After correlation analysis between the TDS and concentration of chlorophyll-a, DOC, TSM, comparing absorption spectra of NPSS, CDOM, chlorophyll-a, and discussion of the remote sensing reflectance in different wave bands, the result shows TDS and water depth are the main influence of IOPs and AOPs. The TDS has influenced the absorption properties remarkably and has high correlation coefficient with the concentration of DOC and with absorption of CDOM in 240-300nm. Water depth is most important factor of remote sensing reflectance, and TDS plays a major role in $550-720 \mathrm{~nm}$ within the same water depth ranges. These conclusions may provide useful RS basis to estimate water quality parameters in desert lakes.

Keywords: Desert lakes; total dissoloved solids; Lake Moon; Tengger Desert; absorption coefficient

我国西北位于干旱、半干旱地区,淡水资源较为缺乏,严重影响和制约了当地居民的正常生活和地方经 济的发展;而经济落后,缺乏资金支持,又限制了当地对水资源的调查和开发,甚至缺少最基本的水文地质 资料. 近年来, 这些地区荒漠化趋势加剧, 吞噬了无数良田和村庄, 由此引起的沙尘暴对北京等重要城市的 空气质量也造成了较大影响, 引起国务院高度重视, 要求切实遏制荒漠化的扩大 ${ }^{[1]}$. 植被种植作为防风固 沙、整治沙漠的最好方法,首先需要解决的也是水的问题, 由此可见供水保障建设是这些地区的第一要务,

* “中国湖泊水质、水量和生物资源调查”专项项目 (2006FY110600) 资助. 2010-03-05 收稿;2010-05-30 收修改 稿. 巫文文,男,1984 年生, 硕士研究生;E-mail:wuman555@163.com. 
而遥感监测技术具有快速高效等特点 ${ }^{[2]}$,可有效加快当地水源信息的普查工作.

我国西北巴丹吉林、腾格里等沙漠中有大量湖泊存在, 是干旱地区不可多得的水源之一. 但由于湖泊成 因和地理环境等原因, 这些湖泊的水质和水体的光学特性与东部太湖等大型湖泊有着较大的差异. 沙漠湖 泊水体的 $\mathrm{COD} 、 \mathrm{BOD}_{5}$ 等主要水质指标较好, 但由于湖泊面积较小, 水深较浅, 蒸发量大, 部分湖水矿化度偏 高, 直接影响水体的光学特性以及限制水体的使用 ${ }^{[3]}$. 现阶段较为成熟的湖泊水质遥感监测模型对沙漠湖 泊无法适用, 且国内外对干旱和沙漠地区湖泊的研究较少, 乔江 ${ }^{[4]}$ 等利用 ETM 图像对腾格里沙漠湖泊及湖 盆绿洲的动态进行了研究; 张辉 ${ }^{[5]}$ 等使用 TM 图像对柴达木盆地中部盐湖环境进行了遥感初步解译; 宋开 山 $^{[6]}$ 等以松嫩平原查干湖为例, 对半干旱地区内陆湖泊透明度进行了估测, 对 TM 图像中水体的光谱特征进 行了分析; 于瑞洪 ${ }^{[7]}$ 等以乌梁素海为例, 对干旱区草型湖泊悬浮物浓度及水深进行了遥感分析; 刘兴起 ${ }^{[8]}$ 等 对吉兰泰盐湖典型地物波谱反射率特征进行了研究, 发现 $550-650 \mathrm{~nm}$ 波段内盐水的反射率大于淡水, 但少 有结合实测光谱, 系统分析沙漠湖泊水体光学特征的研究, 从而限制了对这类地区湖泊遥感工作的深人和 扩展.

本文以腾格里沙漠月亮湖为例, 通过对各种水质参数、吸收系数和水体遥感反射比的研究, 分析高矿化 度沙漠湖泊水体特有的固有和表观光学特性, 讨论水深、矿化度等参数对水体光学特性的影响, 为建立遥感 监测沙漠湖泊矿化度等水质指标的光学模型提供理论支持.

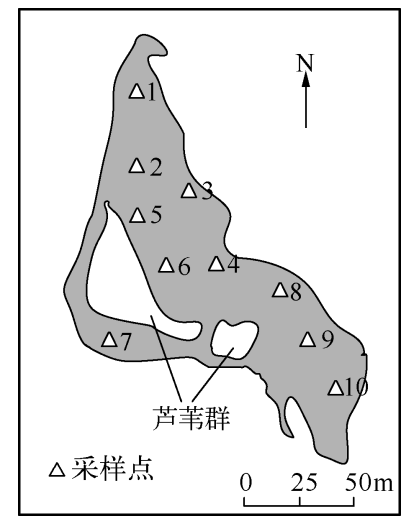

图 1 月亮湖及采样点空间位置

Fig. 1 Sampling positions in Lake Moon

\section{1 数据的获取}

2009 年 9 月 25 日至 10 月 5 日, 深人腾格里沙漠, 对月亮湖、巴 彦浩特生态公园淡水池进行了水体光谱测试和同步水质采样 (图 1). 月亮湖 $\left(38^{\circ} 46^{\prime} \mathrm{N}, 105^{\circ} 15^{\prime} \mathrm{E}\right)$ 为典型的沙漠腹地湖泊, 湖盆之外 为流动沙丘, 湖盆边缘为固定沙丘及半固定沙丘, 伴生有菆芝草、沙 竹、草甸等植物, 湖泊内有淡水补给泉出水头致使其附近生长有只 能在淡水中存在的芦苇群 ${ }^{[9]}$, 湖泊呈长条形, 长轴方向多和主风向 一致, 面积约 $3 \mathrm{~km}^{2}$, 水深很浅, 最深处不超过 $1.8 \mathrm{~m}$, 常年积水, 水质 清澈, 基本未受污染. 由于沙漠中交通极为不便, 携带水样困难, 另 外沙漠湖泊面积较小, 因此在满足研究需要的前提下, 选取了较少 的采样点. 布点首要考虑的是在不同深度、矿化度水体光谱的区别, 其中月亮湖为咸淡水混合湖, 矿化度差异较大, 共采样 10 个点 (1 至 10 号点); 另选择同纬度巴彦浩特生态公园淡水池, 采样 1 个点 (11 号点) 用于对比. 每个点采集 4 瓶水样, 放人装有冰块的保温箱 中, 带回实验室进行水化学参数和固有光学特性分析, 主要参数包括: 叶绿素 $\mathrm{a} 、$ 有机悬浮物 (OSM)、无机悬 浮物 ( ISM) 、溶解性有机碳 (DOC) 、 $\mathrm{pH}$ 值、总硬度、总碱度、溶解性总固体 (TDS). 同时记录了每个采样点监 测时的时间、天气状况、GPS 点位坐标、水温、水深和透明度.

部分参数的测定方法为: 叶绿素 a 含量采用分光光度计法测定, 用 $1.1 \mu \mathrm{m} \mathrm{GF} / \mathrm{C}$ 滤纸过滤, $90 \%$ 热乙醇 提取, 然后用 7230 分光光度计检测, 计算; 悬浮物的测定采用电子天平称重法, 即用灼烧后的 $\mathrm{GF} / \mathrm{C}$ 滤纸过 滤, 烘干, 冷却, 计算; DOC 的含量使用 1020 型 TOC 仪测定; pH 值、总硬度、溶解性总固体使用 GB/T 57492006 规定方法测定; 总碱度使用 SL83-1994 方法测定.

黄色物质、叶绿素 $\mathrm{a}$ 、无机悬浮物、总悬浮物 ( TSM) 的光谱吸收系数测定采用 $0.45 \mu \mathrm{m} \mathrm{GF/F}$ 过滤的水样 先在分光光度计下测定吸光度, 然后根据公式计算和校正, 得到最终结果. 其中黄色物质事先用 $10 \%$ 的盐酸 中浸泡过得 $0.2 \mu \mathrm{m}$ 的聚碳酸脂 (polycarbonate) 滤纸, 在 $120-140 \mathrm{~mm} \mathrm{Hg}$ 的负压下, 过滤水样得到黄色物质 样品, 同时过滤超纯水得到超纯水空白. 样品立即进行分光光度计测量, 分光光度计使用的是岛津 UV-2401, 测量时波长间隔 $1.6 \mathrm{~nm}$, 狭缝宽度 $2 \mathrm{~nm}$, 测量波长范围是 $400-600 \mathrm{~nm}$; 悬浮物用 $\mathrm{GF} / \mathrm{F}$ 玻璃纤维滤纸过滤 $200-500 \mathrm{ml}$ 的水样, 在分光光度计下测定滤膜上颗粒物的吸光度, 用同样湿润程度的空白滤膜做参比, 以 $750 \mathrm{~nm}$ 的吸光度为基准对其他波段进行校正. 
实测光谱采用双通道地物光谱仪 ASDFieldSpec 931 (美国 ASD 公司生产, 光谱 350-1050nm,8 度视场 角观测), 基于 NASA 水体光谱测量规范 ${ }^{[10]}$, 仪器在试验前对两个探头以及标准反射板进行了定标, 并注意 规避了水面白帽和波浪的影响. 数据测量完后回到实验室, 首先在 ASD ViewSpecPro 软件中对数据进行篮 选, 剔除所有数值较高或异常的曲线, 保留数值较低的曲线, 然后把剩余的曲线进行平均, 并以文本格式输 出,再利用输出的结果导人 Excel 计算水体的离水辐亮度和遥感反射率比.

\section{2 水质参数相关性分析}

月亮湖水质较好,透明度很高, 水体偏碱性, $\mathrm{pH}$ 在 $8-9$ 之间,矿化度在 $6-8 \mathrm{~g} / \mathrm{L}$ 之间 (表 1). 与巴彦浩 特生态公园淡水水体相比, 月亮湖水体叶绿素 a 含量很低, 是巴彦浩特生态公园淡水水体的 $1 / 15-1 / 10$, 而 总悬浮物浓度约为其 $1 / 2$, 另外无机悬浮物比例高于有机悬浮物. 需要注意的是月亮湖水体 DOC 含量很高, 为生态公园淡水池的 60-100 倍. 由于月亮湖为承压地下水补给, 造成湖泊水体的矿化度分布不均, 靠近补 给源的区域矿化度较低. 由于最靠近补给区, 7 号点矿化度最低, 为 $6 \mathrm{~g} / \mathrm{L}, 6$ 号和 8 号点矿化度在 $7-8 \mathrm{~g} / \mathrm{L}$ 之 间,其余各点矿化度在 $8 \mathrm{~g} / \mathrm{L}$ 以上,其中 4 号点矿化度最高,达到 $8.5 \mathrm{~g} / \mathrm{L}$.

表 1 部分水质参数

Tab. 1 Water quality parameters of the samples

\begin{tabular}{|c|c|c|c|c|c|c|c|c|c|c|c|}
\hline \multicolumn{2}{|c|}{ 水体 } & $\begin{array}{l}\text { Chl. a } \\
(\mu \mathrm{g} / \mathrm{L})\end{array}$ & $\begin{array}{c}\text { DOC } \\
(\mathrm{mg} / \mathrm{L})\end{array}$ & $\begin{array}{c}\text { TSM } \\
(\mathrm{mg} / \mathrm{L})\end{array}$ & $\begin{array}{c}\text { OSM } \\
(\mathrm{mg} / \mathrm{L})\end{array}$ & $\begin{array}{c}\text { ISM } \\
(\mathrm{mg} / \mathrm{L})\end{array}$ & $\mathrm{pH}$ & $\begin{array}{l}\text { 总硬度 } \\
\text { (mg/L) }\end{array}$ & $\begin{array}{c}\text { TDS } \\
(\mathrm{mg} / \mathrm{L})\end{array}$ & $\begin{array}{l}\text { 水深 } \\
(\mathrm{m})\end{array}$ & $\begin{array}{c}\text { 透明度 } \\
(\mathrm{m})\end{array}$ \\
\hline \multirow[t]{3}{*}{ 月亮湖 } & 最小值 & 0.38 & 182.3 & 17.25 & 3.71 & 11.75 & 7.97 & 1694.9 & 6116.4 & 0.5 & 清澈见底 \\
\hline & 最大值 & 0.78 & 274.8 & 25.67 & 9.25 & 19.67 & 8.40 & 2033.7 & 8491.1 & 1.8 & 1.6 \\
\hline & 平均值 & 0.50 & 260.2 & 20.10 & 6.15 & 13.96 & 8.15 & 1847.8 & 7938.3 & 1.1 & 清澈见底 \\
\hline \multicolumn{2}{|c|}{ 生态公园淡水池 } & 6.44 & 3.6 & 38.00 & 13.00 & 25.00 & 7.50 & 558.0 & 781.1 & 0.5 & 0.4 \\
\hline
\end{tabular}

矿化度过高是沙漠湖泊水体无法用于人畜饮用和植被灌溉的最主要原因,也是沙漠湖泊水体与一般湖 泊最大的区别之一,所以矿化度成为沙漠湖泊研究的重点 ${ }^{[11]}$. 矿化度是水中所含各种离子、分子及化合物 (不包括游离状态的气体) 的总量, 通常是以水烘干后所得残渣来确定, 以 $\mathrm{mg} / \mathrm{L}$ 表示. 总矿化度说明了水中 所含盐量的多少, 表示水的矿化程度, 是水体化学成分的重要标志, 低矿化度的水 (淡水) 常以重碳酸根 $\left(\mathrm{HCO}_{3}^{-}\right)$为主要成分, 中等矿化程度的水常以硫酸根 $\left(\mathrm{SO}_{4}^{2-}\right)$ 为主要成分, 高矿化程度的水以氯离子 $\left(\mathrm{Cl}^{-}\right)$ 为主要成分 ${ }^{[12]}$. 选取月亮湖的 10 个采样点进行矿化度浓度与 DOC、叶绿素 $\mathrm{a} 、$ 总悬浮物浓度和 $\mathrm{pH}$ 值的线性 相关性和显著性分析 (图 2).

矿化度与总悬浮物的相关性最低 $\left(R^{2}=0.0441, P>0.05\right)$; 与 $\mathrm{pH}$ 值相关性为 $0.365, P>0.05$; 与 叶绿素 a 浓度呈负相关 $\left(R^{2}=0.4737, P<0.05\right)$; 与 DOC 相关性最高, 具有显著正相关性 $\left(R^{2}=\right.$ $0.9641, P<0.01)$. 矿化度与叶绿素 $\mathrm{a}$ 的负相关是由于过高的矿化度会阻碍水中浮游植物和藻类的生 长 ${ }^{[13]}$, 同时由于湖泊受人为干扰较少, 水中缺少浮游植物生长所需的磷等营养物, 所以沙漠湖泊中叶 绿素含量低; 而总悬浮物与矿化度无直接联系, 水中盐分并不依附于各种颗粒存在, 所以相关性最小, 但沙漠湖泊周围有大量沙粒等无机颗粒存在,造成沙漠湖泊水体中无机颗粒比例偏高; 由于盐类水解 产生的氢氧根离子是 $\mathrm{pH}$ 的来源之一, 所以矿化度与 $\mathrm{pH}$ 值有一定的联系, 并使水体呈现弱碱性; DOC 代表溶人水中的有机质的含量, 正常状况下有机物较难溶于水中, 所以淡水中的 DOC 含量较少, 但矿 化度较高的水体却可以增加有机物的溶解性, 其原因之一是矿化度水体中阴阳离子的水解反应产生 的氢氧根中和了有机酸等难溶有机物的水解反应产生的氢离子, 对于有机酸的水解反应, 在相同的温 度下, 化学平衡常数 $\lg K$ 是保持不变的,所以为了维持平衡常数,有机酸的水解反应会加剧, 最后达到 一个新的平衡, 从而促进了有机质的溶解 ${ }^{[14]}$. 另一方面由于沙漠水体无法流动, 所有植物死亡分解后, 全部留在水中, 提高了有机质的含量. 可见矿化度在一定程度上都直接或间接影响水体中其它成分的 含量,使沙漠水体的水质参数有别于 11 号淡水水体. 

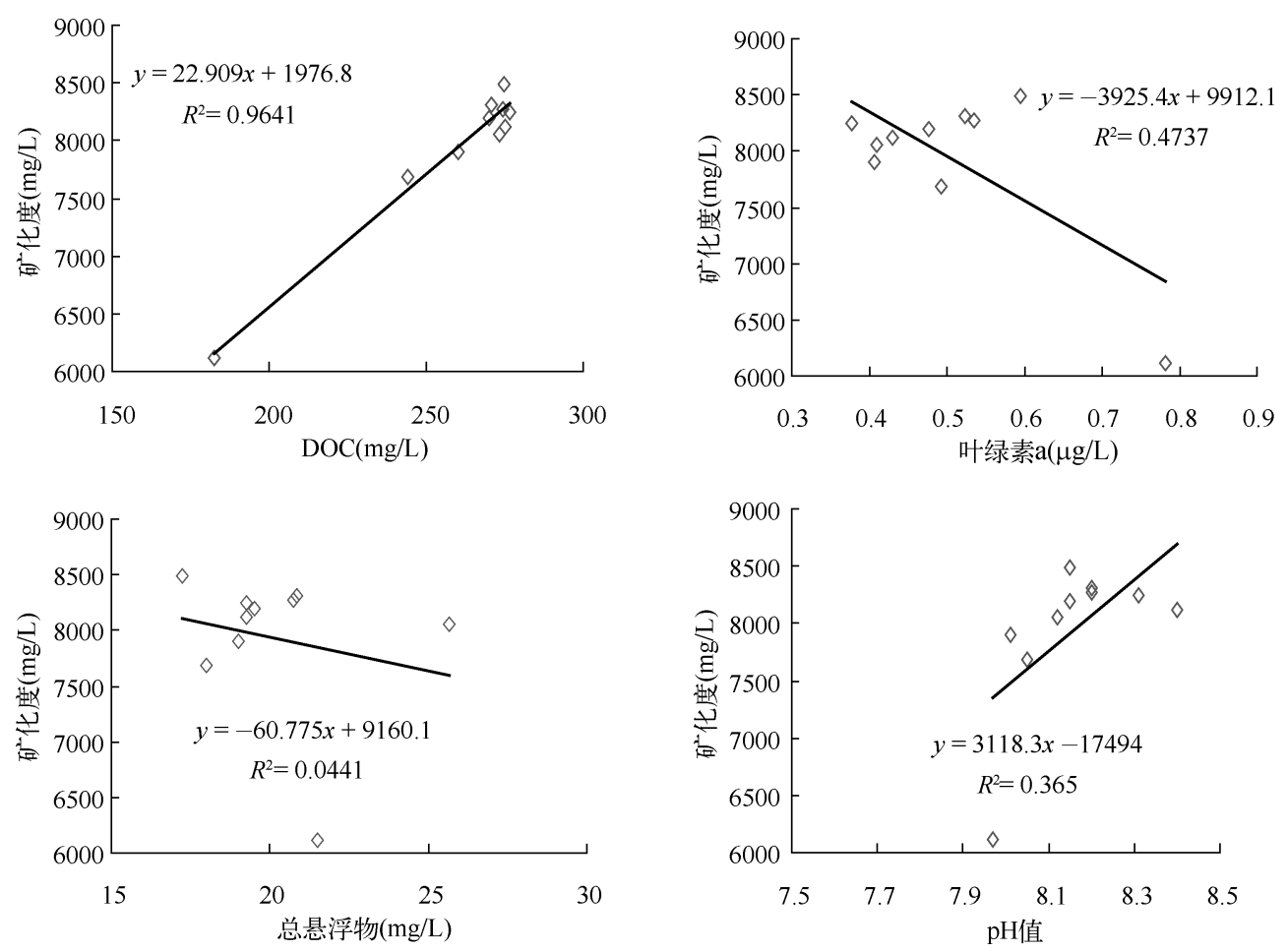

图 2 月亮湖水体矿化度与主要水质参数相关性分析

Fig. 2 Correlative coefficient between TDS and main water quality parameters of Lake Moon

\section{3 月亮湖水体固有光学特性}

\section{1 黄色物质吸收光谱分析}

CDOM 是 DOC 中可吸收光的部分, 可能由氨基酸、糖、氨基糖和脂肪酸类组成; 也可能包含有类夢卜素、 氯纶色素、碳水化合物和酚 ${ }^{[15]}$. CDOM 由于成分复杂, 浓度确定很困难, 最常用方法是用 $355 \mathrm{~nm} 、 375 \mathrm{~nm}$ 和 $440 \mathrm{~nm}$ 等波长处的吸收系数来表示其浓度. 吸收系数越大, 说明对应样点的 CDOM 浓度越高. CDOM 吸收光 谱特性研究中最关注的另一个量值是 $S$, 它反映了黄色物质指数吸收的衰减程度. 吸收系数光谱一般随波长 的增加呈指数衰减 ${ }^{[16]}: a_{\mathrm{g}}(\lambda)=a_{\mathrm{g}}\left(\lambda_{0}\right) \exp \left[-S\left(\lambda_{0}-\lambda\right)\right]$, 其中 $a_{\mathrm{g}}(\lambda)$ 为黄色物质在波长 $\lambda$ 处的吸收系数, $\lambda_{0}$ 是参考波段值, 通常选择 $440 \mathrm{~nm} . a_{\mathrm{g}}\left(\lambda_{0}\right)$ 是参考波段的光吸收系数, $S$ 是吸收系数曲线的指数斜率参数, 这 里采用最小二乘法对 $240-440 \mathrm{~nm}$ 波段进行非线性拟合, 得到指数斜率参数 $S . S$ 的值独立于参考波段 $\lambda_{0}$, 但是会因为地理位置和时间的不同和黄色物质来源不同而有所差异.

生态公园淡水 CDOM 的光谱吸收曲线随波长增加呈指数规律递减, 如图 $3 \mathrm{a}$ 中红色 $\left(a_{\mathrm{g}} 11\right)$ 所示, 在 $240-260 \mathrm{~nm}$ 间下降最快, 之后趋于缓和, 在 $380 \mathrm{~nm}$ 处接近于 0 . 对于月亮湖水体, CDOM 的光谱吸收曲线仍 然随波长增加呈指数规律递减, 但吸收系数在所有波段都明显高于淡水, 随着波长的增长, 差异减小, 曲线 变化的拐点右移至 $320-350 \mathrm{~nm}$ 间, 拐点之前, 吸收系数随矿化度变化明显.

选取矿化度变化较大的 $2 、 5 、 7 、 11$ 号四个采样点, 比较它们的 $a_{\mathrm{g}}$ 和 $S$ 可见波长越短, 吸收系数越高, 与 矿化度的正相关性越强; 淡水的 $S$ 明显大于咸水, 而不同矿化度的咸水 $S$ 变化不大 (表 2). 为了进一步研究 矿化度对吸收光谱的影响, 选取 $240 、 300 、 320 、 375$ 和 440nm 作为控制点, 对这五个节点上月亮湖 1-10 号采 样点水体的吸收系数与 DOC 浓度和矿化度进行指数拟合, 结果表明, DOC 浓度与 $240 、 300 、 320 、 375 、 440 \mathrm{~nm}$ 处黄色物质吸收系数指数拟合 $R^{2}$ 分别为 $0.9291 、 0.6127 、 0.1591 、 0.1695 、 0.150$; 矿化度浓度与 $240 、 300$ 、 $320 、 375 、 440 \mathrm{~nm}$ 处黄色物质吸收系数指数拟合 $R^{2}$ 分别为 $0.9342 、 0.7192 、 0.2574 、 0.0837 、 0.0846$. 
表 2 黄色物质吸收光谱特征参数

Tab. $2 a_{\mathrm{g}}$ and $S$ of CDOM in Lake Moon

\begin{tabular}{cccccc}
\hline 采样点 & 矿化度 $(\mathrm{mg} / \mathrm{L})$ & $a_{\mathrm{g}}(240)\left(\mathrm{m}^{-1}\right)$ & $a_{\mathrm{g}}(375)\left(\mathrm{m}^{-1}\right)$ & $a_{\mathrm{g}}(440)\left(\mathrm{m}^{-1}\right)$ & $S(440)\left(\mathrm{nm}^{-1}\right)$ \\
\hline 11 & 781.03 & 33.55 & 0.4544 & 0.0625 & 0.03097 \\
7 & 6116.49 & 145.72 & 6.1602 & 1.6693 & 0.02122 \\
5 & 7896.41 & 161.90 & 5.6999 & 1.3818 & 0.02280 \\
2 & 8308.23 & 171.51 & 6.1024 & 1.5539 & 0.02228 \\
\hline
\end{tabular}

对于月亮湖水体,DOC 和矿化度在短波段内与黄色物质吸收系数具有较强的相关性,特别是 $240 \mathrm{~nm}$ 处 与黄色物质吸收系数的相关性极高, $300 \mathrm{~nm}$ 后随着波长的增加而急剧下降,之后一直保持在一个较低的水 平. 黄色物质在激发光照射下能发出苂光, 实验表明, 用紫外光激发水体黄色物质产生苂光时, 水体本身也 产生拉曼反射,这主要是由水分子羟基引起的 ${ }^{[17]}$, 而羟基与矿化度浓度和 DOC 浓度有着紧密的联系,因为 两者的水解共同决定了水体羟基的浓度, 从而使紫外光波段黄色物质的吸收与两者有着紧密的联系.

\section{2 浮游植物吸收光谱分析}

淡水浮游植物吸收光谱在 430-440nm、670-690nm 两处存在两个特征吸收峰, 主要是叶绿素 a 在此处 吸收所致 ${ }^{[18]}$, 在 $370-380 \mathrm{~nm} 、 550-560 \mathrm{~nm}$ 处存在两处波谷, 在 $700 \mathrm{~nm}$ 后吸收趋近于 0 , 如图 $3 \mathrm{~b}$ 中红色 $\left(a_{\mathrm{ph}} 11\right)$ 曲线所示. 当水体受到矿化度影响后, 吸收系数随波长变化趋势变缓, 在 $375-390 \mathrm{~nm}$ 之间出现第一 个吸收峰, 但峰值远低于淡水时的水平, 矿化度越低, 峰值越高, $400 \mathrm{~nm}$ 之后趋于平缓, 在 $490-500 \mathrm{~nm}$ 处开 始明显下降, $670-690 \mathrm{~nm}$ 处出现第二个吸收峰, 峰值很小, $700 \mathrm{~nm}$ 后吸收系数再次降低并趋于 0 .

选择两个吸收峰的波长 $375 、 675 \mathrm{~nm}$ 及 $440 、 550 \mathrm{~nm}$ 作为控制点, 对这四个节点上月亮湖 1-10 号采样点 水体的吸收系数与叶绿素 $\mathrm{a}$ 浓度和矿化度进行线性拟合,结果表明, 叶绿素 a 浓度与 $375 、 440 、 550 、 675 \mathrm{~nm}$ 处 浮游植物吸收系数线性拟合 $R^{2}$ 分别为 $0.7404 、 0.7679 、 0.3610 、 0.1593$; 矿化度浓度与 $375 、 440 、 550 、 675 \mathrm{~nm}$ 处浮游植物吸收系数线性拟合 $R^{2}$ 分别为 $0.7035 、 0.7234 、 0.0113 、 0.0318$. 综合浮游植物特征吸收曲线和相 关性分析, 可见在 $375-440 \mathrm{~nm}$ 波段, 矿化度浓度对吸收的影响较大, 致使吸收曲线与淡水有非常大的区别, $440 \mathrm{~nm}$ 之后吸收曲线重新由叶绿素 $\mathrm{a}$ 控制, 只是吸收系数由于矿化度的影响,数值普遍降低,但基本保持了 淡水吸收系数的曲线形状. 月亮湖水体浮游植物吸收系数普遍较低是由于植物生长所需的营养盐的缺乏导 致叶绿素 a 含量降低,而矿化度在紫外光段的相关性可能是由于咸水促进了硅藻等藻类的生长,这些藻类 含有或分泌特殊的强 UV 吸收物质 ${ }^{[19]}$, 如 MAA (My-crosporine-like Amino Acid), 从而导致 375-425nm 间吸 收系数的大幅降低.

\section{3 非藻类悬浮物吸收光谱分析}

非藻类悬浮物包括浮游植物死亡而产生的有机碎屑, 以及陆生或湖体底泥经再悬浮而产生的无机悬浮 颗粒 ${ }^{[20]}$. 非藻类悬浮物的浓度、颗粒大小和矿质组成是影响其吸收特征的主要因素. 从图 4 红色曲线 $\left(a_{\mathrm{d}} 11\right)$ 我们可以发现淡水非藻类悬浮物的吸收光谱大致呈指数下降,在 $750 \mathrm{~nm}$ 后趋近于 0 , 但对于月亮湖水体,受 矿化度影响后, 吸收系数的下降趋势加剧, 由于月亮湖中悬浮物含量小于淡水, 吸收系数总体上小于淡水, 从 $350 \mathrm{~nm}$ 起开始急剧下降, 在 $370-380 \mathrm{~nm}$ 处出现拐点,之后下降趋势变缓, $450-600 \mathrm{~nm}$ 吸收系数很低, 趋 近于 0 .

选取 $350 \mathrm{~nm}$ 、拐点 $370 \mathrm{~nm}$ 和 $440 \mathrm{~nm} 、 550 \mathrm{~nm}$ 作为控制点, 对这四个节点上 9 个采样点水体 (由于数据异 常, 去除 1 号点) 的吸收系数与无机颗粒物浓度和矿化度进行指数拟合, 结果表明, 无机颗粒物浓度与 350 、 $370 、 440 、 550 \mathrm{~nm}$ 处非藻类悬浮物吸收系数指数拟合 $R^{2}$ 分别为 $0.0046 、 0.0559 、 0.0871 、 0.0077$; 有机颗粒物 浓度与 $350 、 370 、 440 、 550 \mathrm{~nm}$ 处非藻类悬浮物吸收系数指数拟合 $R^{2}$ 分别为 $0.3086 、 0.0067 、 0.6966 、 0.1484$; 矿化度浓度与 $350 、 370 、 440 、 550 \mathrm{~nm}$ 处非藻类悬浮物吸收系数指数拟合 $R^{2}$ 分别为 $0.3449 、 0.0596 、 0.314$ 、 0. 0145. 综合分析表明, 虽然无机颗粒物的含量高于有机颗粒物, 但有机颗粒物对吸收系数的影响大于无机 颗粒物, 矿化度在 $350-440 \mathrm{~nm}$ 间与吸收系数联系较为紧密, 在此阶段总体上矿化度越大吸收系数越大, 这 是由于水体中有机碎屑的吸收特征与水体中溶解性有机物的吸收特性很相似 ${ }^{[21]}$, 但由于生态公园淡水的悬 
浮物浓度比月亮湖湖水高出许多, 所以 11 号点的吸收系数大于其它样点. $370 \mathrm{~nm}$ 拐点处吸收系数与各种物 质的相关性都很低.
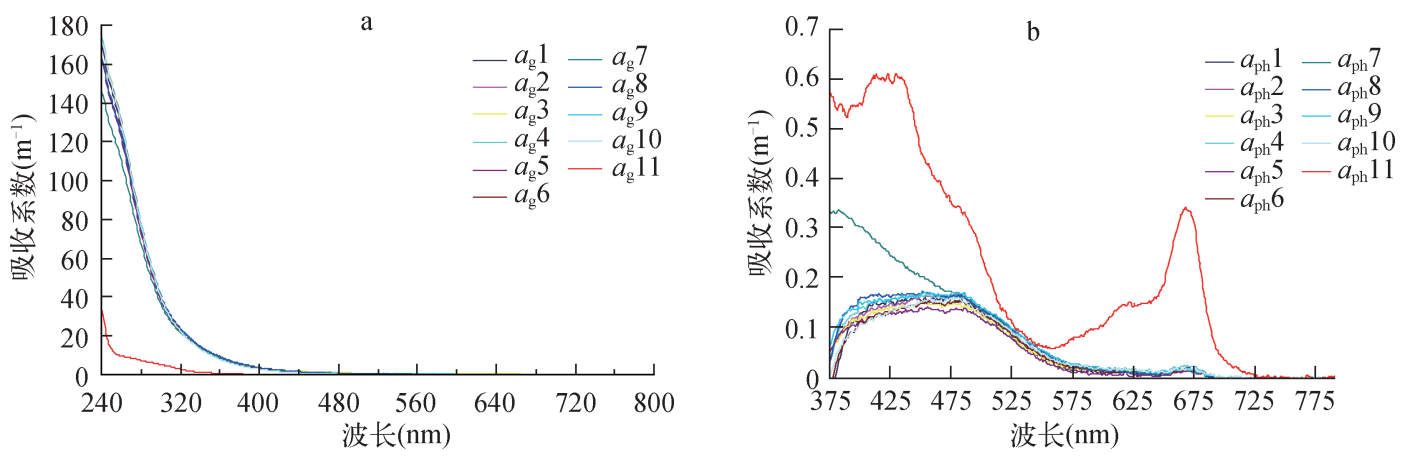

图 3 黄色物质 $(\mathrm{a})$ 和浮游植物 ( b) 吸收光谱

Fig. 3 Absorptions spectra of $\operatorname{CDOM}(\mathrm{a})$ and Chl. a(b)

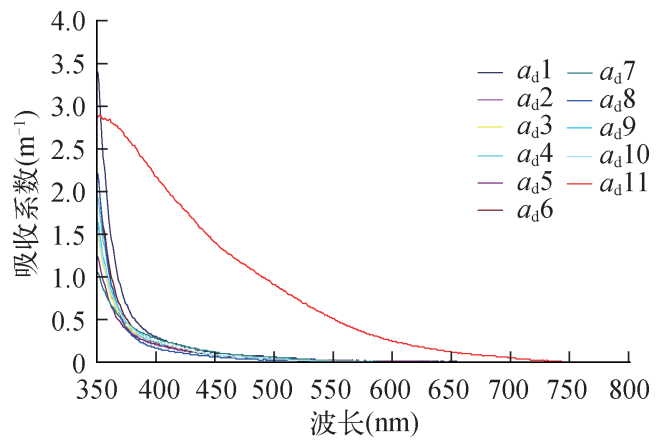

图 4 非藻类悬浮物吸收光谱

Fig. 4 Absorptions spectra of NPSS

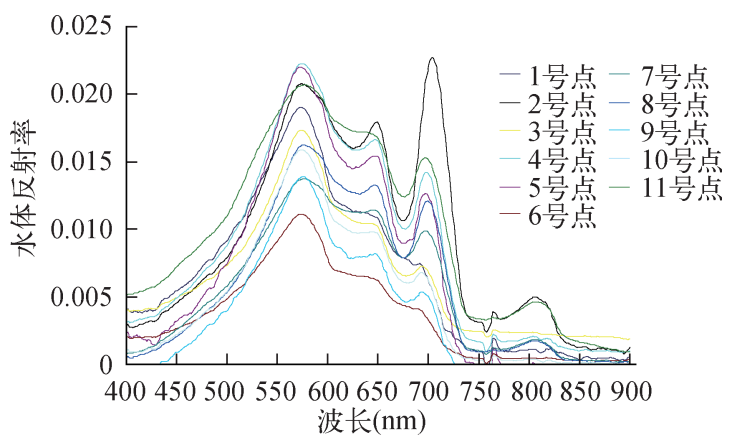

图 5 实测水体光谱反射曲线

Fig. 5 Remote sensing reflectance of Lake Moon

综合以上物质的吸收光谱, 我们不难发现, 沙漠湖泊水体黄色物质的吸收系数远大于 11 号淡水体, 而 浮游植物和非藻类悬浮物的吸收系数略低于 11 号淡水体. 矿化度对沙漠湖泊水体各物质的吸收系数都产 生了显著影响, 特别是在紫外光波段内与黄色物质具有极好的正相关性, 在蓝光波段与浮游植物吸收光谱 存在较好的负相关性. 其中 $240-550 \mathrm{~nm}$ 内黄色物质的吸收系数值远远大于总悬浮物的吸收系数, 倍数在 5 倍以上, 同时此波段内纯水的吸收系数很小, 所以在这一阶段总吸收系数主要体现的是黄色物质吸收光谱 的特征, 因其极强的吸收性而使矿化度越高, 水体反射率越低. Zaneveld JRV 等指出当水中矿化度与淡水差 别较大时, 必须考虑对总吸收系数进行修正, 即 $a_{\mathrm{s}}(\lambda)=a(\lambda)-\psi_{\mathrm{s}}\left(s-s_{\mathrm{t}}\right)^{[22]}$, 其中 $\psi_{\mathrm{s}}$ 为矿化度修正函数, $s_{\mathrm{t}}$ 为参考矿化度, $s$ 为实测矿化度, 修正系数的出现本应使矿化度与吸收系数呈反比, 但通过上述分析可知该 公式应用范围应局限于特定的波段内,在 $240-550 \mathrm{~nm}$ 段并不适用.

\section{4 月亮湖表观光学特性}

对原始光谱数据进行分析, 300-400nm 和 $900-1050 \mathrm{~nm}$ 的光谱不够稳定, 选取 $400-900 \mathrm{~nm}$ 为研究段, 反射光谱特征曲线见图 5 .

月亮湖水体具有典型的内陆水体特征: 在 400-500nm, 由于叶绿素 a 在蓝紫光波段的吸收及黄色物质 在该范围的强烈吸收, 水体的反射率较低 ${ }^{[23]}$; 在 $550-580 \mathrm{~nm}$ 范围的反射峰是由于叶绿素和胡萝卜素弱吸收 和细胞的散射作用形成的 ${ }^{[24-25]}$; 在 $670 \mathrm{~nm}$ 由于叶绿素对红光的吸收出现较低的反射率 ${ }^{[26-27]}$; 700nm 附近反 射峰出现原因是水和叶绿素 a 在该处的吸收系数达到最小 ${ }^{[28-30]}$; 在 $760 \mathrm{~nm}$ 处存在不太显著的曲线起伏, 还 需要进一步地分析其产生原因; $810 \mathrm{~nm}$ 附近的波峰, 与海洋型二类水体吻合, 是颗粒物散射与水体吸收系数 
共同作用的结果. 但月亮湖与普通内陆水体相比具有一些独有的特征:

(1) 月亮湖水体的悬浮物和叶绿素 $\mathrm{a}$ 的含量较内陆一般湖泊小许多,所以反射率较低,但由于月亮湖水 体三大物质的含量低,所以小的浓度变化能够更显著地反映,使反射曲线的起伏大于普通内陆湖泊的水体, 波峰波谷都较一般水体显著和突出, 不同矿化度水体在可见光段反射率相差较大.

(2) 对于含盐水体, 总吸收系数需要进行矿化度修正, 即减去一个与矿化度呈正比的修正函数, 所以矿 化度越高, 总吸收系数越小, 反射率越大 ${ }^{[22]}$. 但在 $400-550 \mathrm{~nm}$ 段, 淡水水体的反射率 (绿色曲线, 11 号采样 点) 却高于其它所有矿化度水体, 这与矿化度越高, 反射率越高的常理相违背. 这与黄色物质在短波段内的 强吸收性有关, 在 $550 \mathrm{~nm}$ 之前一直大于总悬浮物吸收系数, 使总吸收系数基本显示为黄色物质的吸收特性, 可以推断, 这种吸收性超过了该波段内矿化度的修正系数, 甚至水深的影响, 致使高矿化度水体吸收系数反 而变大,淡水的反射率超过含盐水体. 但这一阶段反射率只能反映含盐水体与淡水体的区别,无法分辨矿化 度的高低,因为黄色物质和矿化度的高相关性只能在更短的波长中反映出来.

(3) 沙漠湖泊水深普遍较浅,且水体清澈, 透明度很高, 使水底底质的反射在很大程度上影响了水体的 反射率, 而水深在很大程度上决定了底质的反射程度,但反射率并未严格按照水深不同而增减, 只是同一水 深组别的水体反射率比较接近. $720-900 \mathrm{~nm}$ 间同一水深组别的水体反射率差异减小, 反射曲线相互聚集. 特 别是在 $810 \mathrm{~nm}$ 的反射峰处最为明显,总吸收系数在此波段接近 0 ,水体反射率基本反映的是水深信息. 反射 曲线可分为高中低三组 (图 5), 识别后发现三组曲线分别对应 $0-0.5 \mathrm{~m}(2 、 11$ 号点) 、0.5-1m(4、5、7、8 号 点)、 $1-2 \mathrm{~m}(1 、 3 、 6 、 9 、 10$ 号点 $)$ 三组水深. 其中, $0-0.5 \mathrm{~m}$ 水深分布明显, 水深增加后, 底质影响减小, 反射率 受矿化度影响增大, $0.5-1 \mathrm{~m}$ 和 $1-2 \mathrm{~m}$ 水深的两组划分较为混乱, 光谱反射曲线图中并无明显界限, 但 $0.5-$ $1 \mathrm{~m}$ 组集中在曲线中部, $1-2 \mathrm{~m}$ 组除 3 号点外都处于反射曲线最下方 $(3$ 号点在 $700 \mathrm{~nm}$ 后由于测量时受风速 和阳光干扰数值异常过高).

(4) 550-720nm 段三大物质的吸收成为次要因素,水深的影响超过了矿化度对水体反射率的影响, 成 为水体反射率的决定因素. 但如果分别对 3 组水体进行分析, 可以发现, 同一组别不同水体的反射率却是由 矿化度大小决定的,且不同矿化度间反射率的差值在此波段内也最大. 这种矿化度的影响是由修正函数造 成的, 即矿化度修正函数产生的减少量超过了吸收物质本身的变化幅度, 而低于底质的影响, 在水体的表观 光学特性上表现为矿化度越高,反射率越高.

综上所述, 可以发现除在反射曲线两端外, 水深与反射率都有很强的相关性, 为影响水体遥感反射率的 第一因素, 特别是在 $700 \mathrm{~nm}$ 波段. 由于水深的影响,矿化度、总悬浮物、DOC、叶绿素 a 与水体的相关性都很 低. 对不同组别的水体进行分析, 发现在去除深度影响后, 矿化度在 550-720nm 成为影响水体反射率的主 要因素, 特别是在 $700 \mathrm{~nm}$ 波段最为突出. 可以推测对于沙漠湖泊,水体矿化度修正系数在 $550-720 \mathrm{~nm}$ 波段 内最为适用.

\section{5 结论}

通过实测数据分析发现: 沙漠湖泊水体与生态公园淡水体相比, 矿化度较高, DOC 含量远远大于淡水, 而叶绿素含量偏低; 矿化度显著影响了沙漠湖泊水体的固有光学特性,特别是对黄色物质吸收系数影响极 大, 使其吸收系数远大于淡水和其它物质; 水深为影响沙漠湖泊水体表观光学特性的最主要因素,但水体反 射率并未严格随水深而变化, 而是体现为不同水深组别对反射率的影响, 同一组别中矿化度成为关键因素, 在 550-700nm 段水体反射率随矿化度的增加而增加. 这些研究成果为使用遥感数据估算和监测沙漠湖泊 水质, 特别是矿化度信息, 提供有力的理论支持. 今后为更全面地了解沙漠湖泊水体的光学特性, 深人了解 矿化度与黄色物质的作用机理 ${ }^{[31]}$, 还需对不同矿化度范围和环境下沙漠湖泊进行详细的调查研究, 以求建 立更为合理和适用范围更宽的光学模型.

\section{6 参考文献}

[ 1 ] 王 涛,陈广庭. 中国的沙漠和戈壁.上海:上海科学技术文献出版社, 2008:20.

[ 2 ] Zhang YZ, Jouni P. Application of an empirical neural network to surface water quality estimation in the Gulf of Finland 
usingcombined optical data and microwave data. Remote Sensing of Environment, 2002,81 :327-336.

[ 3 ] 陈善科,吴 平. 腾格里沙漠对周边地区生态环境的影响及其综合治理措施. 草业科学, 2003,20(2):1-3.

[4] 乔 江,王永利. 腾格里沙漠内湖泊及湖盆绿洲的动态研究. 内蒙古气象,2006,2:26-28.

[ 5] 张 辉,韩凤清. 柴达木盆地中部盐湖环境遥感初步解译. 盐湖研究,2002,10(1):28-31.

[6]宋开山, 张 柏, 王宗明等. 半干旱区内陆湖泊透明度高光谱估测模型研究一一松嫩平原查干湖为例. 水科学进 展, 2006, 17 (6) : 790-795.

[ 7 ] 于瑞宏, 刘廷胥, 李畅游等. 干早区草型湖泊悬浮固体浓度及水深的遥感与分析. 水利学报, 2005,36(7):853-862.

[8] 刘兴起,张 辉. 吉兰泰盐湖典型地物波谱反射率特征及其遥感解译标志. 湖泊科学,2000,12(3) :263-266.

[9] 丁宏伟,王贵玲. 巴丹吉林沙漠湖泊形成的机理分析. 干旱区研究,2007,24(1):1-3.

[10] Mueller JL, Austin RW. Ocean optics protocols for SeaWiFS validation. NASA Technical Memorandum 104566. Greenbelt, MD, NASA Goddard Space Flight Center, 1995.

[11] 王跃峰,陈瑞保,白朝军等. 西藏盐湖 TM 影像遥感分析. 盐湖研究,2002,12(2): 1-7.

[12] Wilson WJ, Yueh SH, Dianardo SJ et al. Passive Active L and S band (PALS) microwave sensor for ocean salinity and soil moisture measurements. IEEE Trans Geosci Remote Sensing, 2001 ,39(5) :1039-1048.

[13] Bowers DG, Mitchelson, Jacob EG. Inherent optical properties of the Irish Sea determined from underwater irradiance measurements. Estuar Coast and Shelf Sei, 1996,43:433-447.

[14] 陈林根. 工程化学基础. 北京: 高等教育出版社,2002:125-130.

[15] Prieur L, Sathyendranath S. An optical classification of coastal and oceanic waters based on the specific spectml absorption curves of phytoplankton pigments, dissolved organic matter, and other particulate materials. Limnol \& Oceanogr, 1981, $26: 671-689$.

[16] KalleK. The problem of the gelbstoff in the sea. Oceanogr Mar Biol Aruse Rev, 1996,4:91-104.

[17] Lin MJ. Application of ocean and coast remote sensing. Beijing: Ocean Press, 1991:120-121.

[18] Vos WL, Donze M, Bueteveld H. On the reflectance spectrum of algae in water: the nature of the peak at $700 \mathrm{~nm}$ and its shift with varying concentration. Technical Report Communication on Sanitary Engineering and water Management, Delft, The Netherlands, $1986: 86-22$.

[19] Rozema J, Bjrn O, Bornman JF et al. The role of UV-B radiation in aquatic and terrestrial ecosystems-an experimental and functional analysis of the evolution of UV-absorbing compounds. Photochem Photobiol B: Biol, 2002 ,66:2-12.

[20] Morrow JH, Chamberlin WS. A two-component description of spectral absorption by marine particles. Limnology Oceanography, $1989, \mathbf{3 4}: 1500$.

[21] Doxaran D, Froidefond JM, Lavender S. Spectral signature of highly turbid waters application with SPOT data to quantify suspended particulate matter concentrations. Remote Sensing of Environment, 2002,81 :149-161.

[22] Zaneveld JRV, Kitchen JC, Moore CC et al. Scattering error correction of reflection-tube absorption meters. Proceedings of SPIE, $1994,2258: 44-55$.

[23] 刘堂友,匡定波, 尹 球. 湖泊藻类叶绿素 $\mathrm{a}$ 和悬浮物浓度的高光谱定量遥感模型研究. 红外与毫米波学报,2004, $23(1): 11-15$.

[24] Gitelson AA, Garbuzov G, Szilagyi F et al. Quantitative remote sensing methods for real-time monitoring of inland waters quality. Int J Remote Sensing, $1993,14(7): 1269-1295$.

[25] 周 艺,周伟奇, 王世新等. 遥感技术在内陆水体水质监测中的应用. 水科学进展, 2004,15(3):312-317.

[26] Zhang YL, Zhang B, Wang X et al. A study of absorption characteristics of chromophoric dissolved organic matter and particles in Lake Taihu, China. Hydrobiologia, 2007,592:105-120.

[27] 李素菊, 吴 情,王学军等. 巢湖浮游植物叶绿素含量与反射光谱特征的关系. 湖泊科学, 2002,14(3):228-234.

[28] Gitelson AA. The peak near 700nm on radiance spectra of algae and water relationship of its magnitude and position with chlorophyll concentration. Int J Remote Sensing, $1992, \mathbf{1 3}: 3367-3373$.

[29] Gordon HR. Diffusive reflectance of the ocean: the theory of its augmentation by chlorophyll-a fluorescence at $685 \mathrm{~nm}$. Applied Optics, 1979,18:1161-1166.

[30 ] Grniger A, Sinha RP. Photoprotective compounds in cyanobacteria, phytoplankton and maeroalgaea database. Photochem Photobiol B: Biol, $2000, \mathbf{5 8}: 115-122$.

[31] 沈 红,赵东至,付云娜等. 黄色物质光学特性及遥感研究进展. 遥感学报,2006,10(6):950-954. 